\title{
QUANTITATIVE INTERPRETATION OF MAPS OF MAGNETIC AND GRAVITATIONAL ANOMALIES BY MATHEMATICAL METHODS*
}

\author{
BY \\ E. G. KOGBETLIANTZ \\ Lehigh University**
}

1. Introduction. In geophysical prospecting for oil and other minerals gravitational and magnetic anomalies corresponding to geological phenomena are mapped. The problem of the quantitative interpretation of such empirical maps consists in the determination of numerical values for all the geological parameters (depth, thickness, slope, density, intensity and direction of magnetization, etc.) which characterize a tectonic structure or an ore-body. To illustrate the possibility of such an interpretation it is preferable to avoid the complications involved in the mathematical study of maps of complex anomalies. The complex anomalies are due to the coexistence in the same region of many different geological phenomena. Resulting from the superposition of many simple anomalies, they can be resolved into their simple components each of which corresponds to a single ore-body or tectonic structure. This resolution is the first step which must be performed, since no interpretation of a complex anomaly map as such is possible. The problem of resolution is a very important one since in most cases we have to deal with complex anomalies, the simple ones being exceptions. Special methods devised by the author solve this important problem, but they are not discussed in this paper which deals with the quantitative interpretation of a simple anomaly map. We study here two cases: an axial anomaly created by an anticline and a centered anomaly corresponding to a salt dome. They are sufficiently simple and at the same time have great practical importance.

A new method of interpretation, based as the usual methods on the theory of potential but essentially different from them, is introduced in this paper. In the usual methods ${ }^{1}$ systematic use is made of individual values such as maxima, minima, zeros, inflection points, etc., of the observed and plotted quantity as well as of their distances. The use of such remarkable values and distances is founded on the tacit assumption that they reflect exclusively the physical action of the unknown structure or ore-body whose study is the object of the interpretation. This assumption is permissible for the anomalies of large magnitude but it is doubtful for those of average magnitude and completely wrong for small anomalies.

In the past, geophysical prospecting by gravitational and magnetic methods was directed mostly toward the study of important, clearly pronounced anomalies of large magnitude which correspond to more shallow deposits or to big well defined tectonic structures. But now the geophysicists are obliged to deal with more difficult

* Received April 11, 1944.

** On leave of absence. Now at The New School, New York.

1 For examples of these usual methods see L. L. Nettleton, Geophysical prospecting for oil, McGrawHill Book Co., New York, 1940, Chapters 6 and 12, and also H. Shaw, Interpretation of gravitational anomalies, Trans. Amer. Inst. Min. Met. Eng. 97, 271-366 (1932). 
cases and they have to interpret maps of small anomalies. Thus, we must study the obstacles which naturally lead to an erroneous interpretation of an anomaly map by the usual methods of remarkable values and distances if the magnitude of the anomaly is not very large.

2. Punctual anomaly. An anomaly map is plotted on the basis of measurements made at isolated stations. It is supposed to be generally correct, and the usual corrections required by the topography of the region, the fact that the earth is not a sphere, etc., are supposed to have already been made. We must emphasize that a quantitative interpretation presupposes an accurate correction for the so-called regional anomaly, and it cannot be expected to yield good results if the latter correction is made, as is customarily (and very unfortunately) done, simply by smoothing arbitrarily the experimental curves. Special methods exist which ensure a very accurate correction for regional anomaly by deducing it from the map itself, but this important question cannot be discussed here. All usual corrections having been made, each individual value obtained at a station is the combined effect of two anomalies: 1) the anomaly caused by the tectonic structure or the ore-body the study of which is the purpose of the interpretation, and 2) the anomaly generated by local irregularities of mass distribution or of magnetization intensity in the immediate vicinity of and under the point of measurement. This strictly local anomaly-we propose to call it "punctual anomaly" - is in general very small. It affects only a small area around the point and it is precisely this punctual anomaly which is responsible for perceptible variations in the value of the observed quantity which occur for small displacements of the apparatus used around the station. In fact the apparatus used now are extremely sensitive and we cannot neglect any more the existence of punctual anomalies. There is no correction at all for them since the punctual anomalies, affecting every observed individual value, cannot be evaluated. If the magnitude of the studied anomaly is large, the punctual anomalies are negligible and the map can be interpreted with the aid of remarkable values and distances. The positive results achieved by the old interpretation methods must be explained in this way. But, if the magnitude of the anomaly is small, the punctual anomalies not only modify the extremal values but they also displace them, altering all the distances used in the usual interpretation methods. Since these old methods express all geological parameters in terms of remarkable values, their distances and ratios, it is plain that punctual anomalies render these methods completely useless in the interpretation of small anomalies. It is a very important though often disregarded fact that the interpretation based on isolated values can in general be only qualitative and gives exactly nothing in case of small anomalies. This important fact explains the lack of success in dealing with maps of small anomalies and is the reason for the actual ineffectiveness of geophysical prospecting in discovering new oilfields in U.S.A. New methods of interpretation well adapted to small anomalies are now necessary. They must be introduced into practice if the geophysical prospecting by gravitational and magnetic methods is to be applied in the future.

The interpretation errors caused by punctual anomalies can and must be eliminated and there is only one possible way to do it. Considered together, the punctual anomalies in the region covered by the measurements have a random distribution; they oscillate about zero and are independent one from another. Consequently, they must undergo an almost total compensation if we form the average value of some 
function of the mapped quantity, the average value with respect to the whole map, using all the observed values at a time. We can eliminate the harmful influence of punctual anomalies and compensate at the same time for the possible residual observation errors only by combining all observed values in an integral. For any map in general the average values express much better the action of the phenomenon under study than do the individual values and their distances. In other words, interpretation rules and formulae based on the systematic use of integrals give much more correct quantitative results in all cases and for all maps. Rules based on special individual values hold only in very rare and exceptional cases of big structures such as, for instance, the shallow salt domes of Texas, the Kursk and Kirunawaara iron ore-bodies or the Great Rhodesian Dyke.

The method described in this paper uses exclusively the average values and, in particular, moment functions and moments of the observed quantity and of its square. This method was applied by the author in France and in Iran with good results. The cases studied here are chosen only for the sake of brevity. The method is elaborated for the most general cases of complex anomaly maps obtained as result of magnetic or gravimetric survey of a completely unexplored region.

3. Center of gravity and first moments. The problem of locating the center of gravity $C$ of disturbing masses is a fundamental one, and its solution is the first step of every interpretation. We solve it in the general cases of an axial anomaly and of a centered anomaly. The coordinates $x^{*}, y^{*}, z^{*}$ of $C$ are expressed with the aid of moments of the observed quantity $Q$, that is in terms of

$$
Q_{m}=\int_{-\infty}^{\infty} x^{m} Q(x) d x
$$

for an axial anomaly and in terms of

$Q_{m n}=\iint_{P} x^{m} y^{n} Q(x, y) d S, \quad Q_{m}=\iint_{P} r^{m} Q(r, \phi) d S, \quad\left(r^{2}=x^{2}+y^{2}, x \tan \phi=y\right)$

for a centered anomaly, the double integration being extended over the infinite plane $x O y$ denoted by $P$.

Axial anomaly. If the geologic feature being considered is much longer in one dimension (strike, axis of anomaly), the corrected map of the axial anomaly created by such a structure is a family of nearly parallel lines and the interpretation deals with a curve describing the behaviour of the plotted quantity on a typical profile perpendicular to the anomaly axis. Cartesian coordinates $x, y, z$ are introduced with the $z$-axis directed vertically downward, the origin $O$ being at the surface and the $x$-axis being perpendicular (the $y$-axis being parallel) to the anomaly axis, as shown in Fig. 1 . The excess of the density of disturbing masses over the density of their environment is called the density-contrast and is denoted by $\sigma(\sigma \lessgtr 0)$. We represent the disturbing structure as a homogeneous cylindrical body of normal cross section $S$, denoting the area of $S$ by $A$. At a point $(x, z)$ in the plane $y=0$ the potential $U$ of the body is given by

$$
U(x, z)=-\frac{1}{2} k \iint_{S} \log \left[(x-\xi)^{2}+(z-\zeta)^{2}\right] d S+\text { const., }
$$

where $\xi, \zeta$ are running coordinates on $S$ and $k=2 f \sigma, f$ being the gravitational constant 
$\left(66.7 \times 10^{-9} \mathrm{cgs}\right.$.) and $\sigma$ the density-contrast. On the surface (plane $\left.P\right), z=0$, the partial derivatives $U_{x}$ and $U_{z}=D g$ of $U$ are given by

$$
U_{x}-i D g=-k \iint_{S}(x-\rho)^{-1} d S=k \oint_{\Gamma} \log (x-\rho) d \zeta,
$$

where $\rho=\xi+i \zeta$ and $\Gamma$ is the boundary of $S$. The second derivatives $U_{x x}$ and $U_{x z}$ are called the curvature $K$ and the gradient $G$ respectively: $K=U_{x x}, G=U_{x \varepsilon}$. Thus

$$
K-i G=k \iint_{S}(x-\rho)^{-2} d S=k \oint_{\Gamma}(x-\rho)^{-1} d \zeta .
$$

Using in (2), (3) the binomial expansion, we deduce for large $|x|$ the approximations which hold for any form of the section $S$. Their first terms for instance are

$$
U_{x} \sim-k A x^{-1}, \quad D g \sim k A z^{*} x^{-2}, \quad G \sim-2 k A z^{*} x^{-3}, \quad K \sim k A x^{-2},
$$

where $z^{*}$ is the depth of the center of gravity $C$. Denoting an arbitrarily chosen origin of the coordinate $x^{\prime}$ on the profile by $O$, we regard the function $D g=D g\left(x^{\prime}\right)$ as known from the measurements. To find $x^{*}=O O^{*}, z^{*}=O^{*} C$ we shall use the first three moments of $D g$,

$$
\begin{gathered}
M_{0}=\int_{-\infty}^{\infty} D g\left(x^{\prime}\right) d x^{\prime}=\pi k A, \quad M_{1}=\int_{-\infty}^{\infty} x^{\prime} D g\left(x^{\prime}\right) d x^{\prime}=\pi k A x^{*}, \\
\int_{-\infty}^{\infty}\left[x^{2} D g(x)-k A z^{*}\right] d x=\pi k \iint_{S}\left(\xi^{2}-\zeta^{2}\right) d S,
\end{gathered}
$$

where in (6) the origin of the coordinate $x$ is the point $O^{*}$, the projection of $C$ on the profile, $x^{*}$ being considered as already found with the aid of (5). In fact, from (2) we deduce that

$$
x^{\prime} U_{x}+k A-i x^{\prime} D g\left(x^{\prime}\right)=k \iint_{S}\left(\rho-x^{\prime}\right)^{-1} \rho d S .
$$

Integrating (2) and (7) with respect to $x^{\prime}$ in $(-\infty, \infty)$ and observing that the integral of $\left(x^{\prime}-\rho\right)^{-1} d x^{\prime}$ equals $i \pi$ since $\zeta$ in $\rho=\xi+i \zeta$ is positive, we have (5). To prove (6) we integrate in $(-\infty, \infty)$ for $x^{*}=0$ the relation

$$
x\left(x U_{x}+k A\right)-i\left(x^{2} D g(x)-k A z^{*}\right)=k \iint_{S}(\rho-x)^{-1} \rho^{2} d S
$$

and compare the coefficients of imaginary terms.

From (5) we deduce the rule $x^{*}=M_{1} / M_{0}$. However, in practice the integration can be carried out only over a finite interval $(-R, R)$, where the known length $R$ is at least four or five times the depth $z^{*}$ of $C$. The contributions from the intervals $(-\infty,-R),(R, \infty)$ can be computed by means of the expansion of $D g(x)$ in powers of $x^{-1}$,

$$
D g(x)=k A z^{*} x^{-2}\left\{1+2 c_{11} z^{*} x^{-1}+\left(3 c_{21}-c_{03}\right) z^{* 2} x^{-2}+0\left(x^{-3}\right)\right\},
$$

where the constants $c_{m n}$ are given by

$$
\left(z^{*}\right)^{m+n} A c_{m n}=\iint_{S} \xi^{m} \zeta^{n} d S .
$$


In general, unless $S$ is very irregular, we have $z^{*} c_{11} \doteqdot x^{*}, z^{* 2} c_{21} \doteqdot x^{* 2}, c_{03} \doteqdot 1$, whence (8) takes the form

$$
D g(x) \doteqdot k A z^{*} x^{-2}\left\{1+2 x^{*} x^{-1}+\left(3 x^{* 2}-z^{* 2}\right) x^{-2}+0\left(x^{-3}\right)\right\} .
$$

If $M_{0}^{*}$ and $M_{1}^{*}$ denote moments computed for the interval $(-R, R)$, i.e.,

$$
M_{0}^{*}=\int_{-R}^{R} D g\left(x^{\prime}\right) d x^{\prime}, \quad M_{1}^{*}=\int_{-R}^{R} x^{\prime} D g\left(x^{\prime}\right) d x^{\prime},
$$

and if we neglect terms of the relative order $O\left\{\left(z^{*} / R\right)^{2}\right\}$, we find that

whence

$$
\pi k A=M_{0}=\left(1+\frac{2 z^{*}}{\pi R}\right) M_{0}^{2}, \quad M_{1}=\left(1+\frac{4 z^{*}}{\pi R}\right) M_{1}^{*},
$$

$$
x^{*}=\left(1+\frac{2 z^{*}}{\pi R}\right) \frac{M_{1}^{*}}{M_{0}^{*}} .
$$

$M_{0}^{*}$ and $M_{1}^{*}$ can be obtained from the experimental curve for $D g\left(x^{\prime}\right)$ by means of a planimeter. From (11), we note that $M_{1}^{*} / M_{0}^{*}$ is a first approximation for $x^{*}$.

If we set $x^{*}=0$, by (9) we easily see that the contribution from the intervals $(-\infty,-R),(R, \infty)$ to the integral on the left side of $(6)$ is of order $\left(z^{*} / R\right)^{2}$. If we neglect terms of order $\left(z^{*} / R\right)^{2}$, (6) can be written in the form

$$
\frac{\pi}{2 R} M_{2}^{*}=M_{0} z^{*}+\frac{k \pi^{2}}{2 R} \iint_{S}\left(\xi^{2}-\zeta^{2}\right) d S,
$$

where, in the usual notation,

$$
M_{2}^{*}=\int_{-R}^{R} x^{2} D g(x) d x .
$$

If we consider only those cases in which the horizontal dimension of $S$ is much smaller than its depth, then $\iint_{S}\left(\xi^{2}-\zeta^{2}\right) d S \doteqdot-z^{* 2} A$. Using this, and substituting for $M_{0}$ from (10), we have

$z^{*}=\frac{\pi M_{2}^{*}}{2 R M_{0}^{*}}\left[1+\frac{\left(\pi^{2}-4\right) z^{*}}{2 \pi R}\right]$

Equations (11) and (12) permit us to compute $x^{*}$ and $z^{*}$ by successive approximations, the successive values being denoted by $x_{n}^{*}, z_{n}^{*}$ $(n=1,2,3, \cdots)$ and the corresponding positions of $O^{*}$ (Fig. 1) by $O_{n}^{*}$. The steps are as follows: (a) We choose a position for $O$ and compute $M_{0}{ }^{*}, M_{1}^{*}$, integrating over the interval $-R>x^{\prime}<R$ with a planimeter. (b) We obtain $x_{1}^{*}$ by setting $z^{*}=0$ in (11) and plot $O_{1}^{*}$. (c) We compute $M_{2}^{*}$

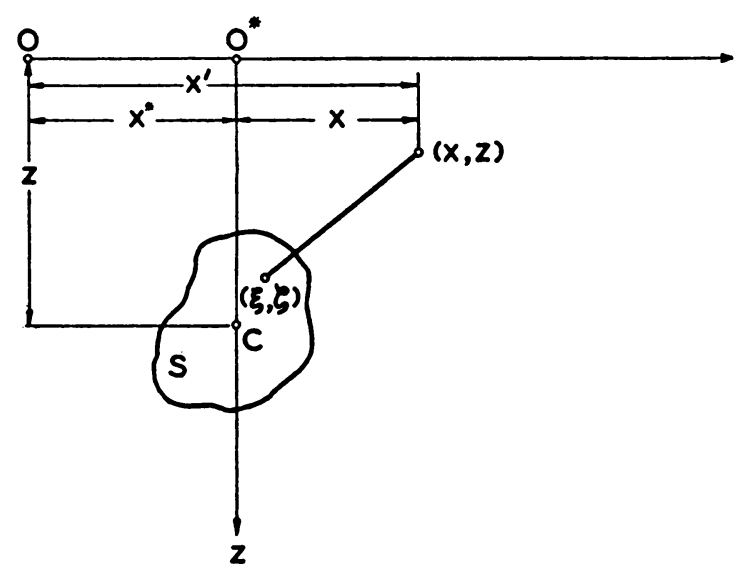

Fig. 1. 
and new values of $M_{0}^{*}$ and $M_{1}^{*}$. (d) Using (12) with $z^{*}=0$ in the right member we compute $z_{1}^{*}$. (e) Using the values of $M_{0}^{*}, M_{1}^{*}, M_{2}^{*}$ obtained in (c), and replacing $z^{*}$ in the right members of (11) and (12) by $z_{1}^{*}$, we obtain $x_{2}^{*}, z_{2}^{*}$ and plot $O_{2}^{*}$. The steps (c) and (e) are repeated until a stabilization of the points $0_{n}^{*}$ and the values $x_{n}^{*}, z_{n}^{*}$ is reached.

The same method can be applied to maps obtained by means of a torsion-balance, giving the curves of the gradient $G(x)$ and the curvature $K(x)$. Denoting the $n$th moment of $G$ by $H_{n}$, we have $H_{0}=0$. Also, integration by parts yields $H_{1}=-M_{0}$, $H_{2}=-2 M_{1}, H_{3}=-3 M_{2}$. For the corresponding reduced moments we have $M_{0}^{*}=$ $-H_{1}^{*}\left(1+2 z^{*} \pi^{-1} R^{-1}\right), 2 M_{1}^{*}=-H_{2}^{*}\left(1+2 z^{*} / \pi R\right), 3 M_{2}^{*}=-H_{3}^{*}+2 k A R z^{*}$. Thus (11), (12) can be transformed into

$$
x^{*}=\frac{H_{2}^{*}}{2 H_{1}^{*}}\left(1+\frac{2 z^{*}}{\pi R}\right), \quad z^{*}=\frac{\lambda H_{3}^{*}}{R H_{1}^{*}}\left(1+\frac{\mu z^{*}}{R}\right),
$$

where $\lambda=\frac{1}{2} \pi /(3 \pi-1)=0.187, \mu=\frac{1}{2}\left(3 \pi^{2}-24 \pi+8\right) /\left(3 \pi^{2}-\pi\right)=0.485$. Equations permit us to determine the center of gravity $C$ from the gradient map only.

The first two moments $L_{0}$ and $L_{1}$ of the curvature $K$ vanish. Since for $|x|$ very large $K(x) \sim k A x^{-2}$, we define the moment $L_{2}$ and the reduced moment $L_{2}^{*}$ by the integrals

$$
L_{2}=\int_{-\infty}^{\infty}\left(x^{2} K-\frac{H_{1}}{\pi}\right) d x, \quad L_{2}^{*}=\int_{-R}^{R}\left(x^{2} K-\frac{H_{1}^{*}}{\pi}\right) d x .
$$

If we multiply (3) by $x^{2}$, subtract $k A$ from both sides and integrate over $(-\infty, \infty)$, we find that $L_{2}=2 H_{1} z^{*}$, the contribution from the intervals $(-\infty,-R),(R, \infty)$ being $-6 k A z^{* 2} / R$. Since $H_{1}=H_{1}^{*}\left(1+4 z^{*} / \pi R\right)$, we then have for $z^{*}$

$$
H_{1}^{*} z^{*}=\alpha L_{2}^{*}\left(1+\beta z /^{*} R\right),
$$

where $\alpha=\frac{1}{2} \pi^{2} /\left(\pi^{2}-4\right)=0.84, \beta=\pi /\left(\pi^{2}-4\right)=0.535$. Equation (14) supplies a control on Eqs. (13).

The magnetic anomaly created by a cylindrical body of section $S$ is related to the gravitational anomaly generated by the same body, and the equations relating to maps of $G$ and $K$ can be transformed into equations relating to maps of the horizontal and vertical components $X$ and $Z$ of the abnormal magnetic field created by the body. If $I$ and $\psi$ denote the magnitude and inclination of the magnetization vector, we have the classical relation (Poisson)

$$
k(X+i Z)=2 I(K+i G) e^{-i \psi},
$$

where $k=2 f \sigma, f$ being the constant of gravitation and $\sigma$ the density-contrast. Multiplying (15) by $x$ and integrating over the interval $(-R, R)$, we obtain for the reduced moments $X_{1}^{*}, Z_{1}^{*}$ the relation $k\left(X_{1}^{*}+i Z_{1}^{*}\right)=2 I\left(L_{1}^{*}+i H_{1}^{*}\right) e^{-i \downarrow}$. Since $L_{1}=0$, we easily find that $\pi R L_{1}^{*}=4 x^{*} H_{1}^{*}\left(1+4 z^{*} / \pi R\right)$. Thus

$$
k X_{1}^{*}=2 I H_{1}^{*}\left(\sin \psi+\frac{4 x^{*}}{\pi R} \cos \psi\right), \quad k Z_{1}^{*}=2 I H_{1}^{*}\left(\cos \psi-\frac{4 x^{*}}{\pi R} \sin \psi\right),
$$

whence we obtain for the two parameters $I A$ and $\psi$,

$$
I A=-\frac{I H_{1}^{*}}{k \pi}=\frac{1}{2 \pi}\left(X_{1}^{* 2}+Z_{1}^{* 2}\right)^{1 / 2}, \quad Z_{1}^{*} \tan \psi=X_{1}^{*}\left(1-\frac{c x^{*}}{R}\right),
$$


where $c$ is defined by the relation $\pi X_{1}^{*} Z_{1}^{*} c=4\left(X_{1}^{* 2}+Z_{1}^{* 2}\right)$. To find $x^{*}, z^{*}$ we need second moments. Since the principal term of the right side of (15) involves $K$, and $K \sim k A x^{-2}$, for large $|x|$ we have $\pi X \sim Z_{1}^{*} x^{-2}, \pi Z \sim-X_{1}^{*} x^{-2}$. Therefore we define the second reduced moments $X_{2}^{*}, Z_{2}^{*}$ by the integrals

$$
X_{2}=\int_{-R}^{R}\left(x^{2} X-Z_{1}^{*} \pi^{-1}\right) d x, \quad Z_{2}^{*}=\int_{-R}^{R}\left(x^{2} Z+X_{1}^{*} \pi^{-1}\right) d x .
$$

Their numerical values can be obtained from the experimental data as areas under curves deduced from the curves $X=X(x), Z=Z(x)$.

Integrating (15) after multiplication by $x^{2}$, and using the defintions of $X_{2}^{*}, Z_{2}^{*}, L_{2}^{*}$, we obtain

$$
k\left(X_{2}^{*}+i Z_{2}^{*}\right)=2 I e^{-i \psi}\left[L_{2}^{*}+i\left(H_{2}^{*}-2 R L_{1}^{*} \pi^{-1}\right)\right] .
$$

Substituting in this result the values of $H_{2}^{*}, L_{2}^{*}$ obtained by solving (13) and (14), and using the relation $2 \pi R L_{1}^{*}=8 x^{*} H_{1}^{*}\left(1+4 z^{*} / \pi R\right)$, we find that

$$
x^{*}=\alpha\left(1+\gamma z^{*} R^{-1}\right) N_{x}, \quad z^{*}=\alpha\left(1+\beta z^{*} R^{-1}\right) N_{z}+4 x^{* 2} \pi^{-1} R^{-1},
$$

where $\alpha$ and $\beta$ are as in (12), $\gamma=2\left(16-\pi^{2}\right) /\left(\pi^{3}-4 \pi\right)=0.665$, and

$$
N_{x}=\left(X_{1}^{*} X_{2}^{*}+Z_{1}^{*} Z_{2}^{*}\right)\left(X_{1}^{* 2}+Z_{1}^{* 2}\right)^{-1}, \quad N_{z}=\left(X_{2}^{*} Z_{1}^{*}-X_{1}^{*} Z_{2}^{*}\right)\left(X_{1}^{* 2}+Z_{1}^{* 2}\right)^{-1} \text {. }
$$

The numbers $N_{x}, N_{z}$ can be deduced from the maps. Thus the moments of $X, Z$ give the four parameters $I A, \psi, x^{*}, z^{*}$.

It is to be noted that the above results can be obtained with the aid of the theory of Fourier transforms. All our results are based on (2), which can be written as a Fourier transform. Now $\rho=\xi+i \zeta$, and since $\min .(\zeta)>0$, we have

$$
i(\rho-x)^{-1}=\int_{0}^{\infty} e^{i t(\rho-x)} d t=\int_{-\infty}^{0} e^{-i t(\rho-x)} d t .
$$

This proves that $D g+i U_{x}$ is the Fourier transform of a function $\omega(t)$, vanishing for positive $t$ and defined in the interval $(-\infty, 0)$ by the relation $\omega(t)=k(2 \pi)^{1 / 2} \iint_{S} e^{-i \rho t} d S$. On the other hand, $D g-i U_{x}$ is the transform of $\overline{\omega(-t)}$, which vanishes for negative $t$, and $D g(x)$ appears as the transform of the function $f(t)$ defined for all values of $t$ by

$$
\begin{aligned}
f(t) & =k\left(\frac{1}{2} \pi\right)^{1 / 2} \iint_{S} e^{i \xi t-\zeta|t|} d S ; \\
D g(x) & =(2 \pi)^{-1 / 2} \int_{-\infty}^{\infty} e^{i x t} f(t) d t .
\end{aligned}
$$

This expression will enable us to find easily the moments of $D g$. The moments of the square of $D g$, which will be required presently can also be deduced easily from (17) with the aid of the Parseval theorem.

Centered anomalies. In the case of a centered anomaly, we represent the disturbing structure as a homogeneous irregular body $B$. Cartesian coordinates $x, y, z$ are introduced, with the $z$-axis directed vertically downward, the origin $O$ being arbitrarily chosen on the plane $P$ (Fig. 2). $C\left(x^{*}, y^{*}, z^{*}\right)$ is the center of mass of $B$, and $O^{*}$ is its projection on the plane $P$ of the map.

The gravitational anomaly is $D g(x, y)$. In the old methods, $O^{*}$ is placed at the 
maximum of $D g$. If the body is a solid of revolution with a vertical axis, this is correct; but if the body is irregular or inclined, the maximum of $D g$ occurs somewhere above its uppermost part. In the present paper, we shall locate $C$ by means of integrals.

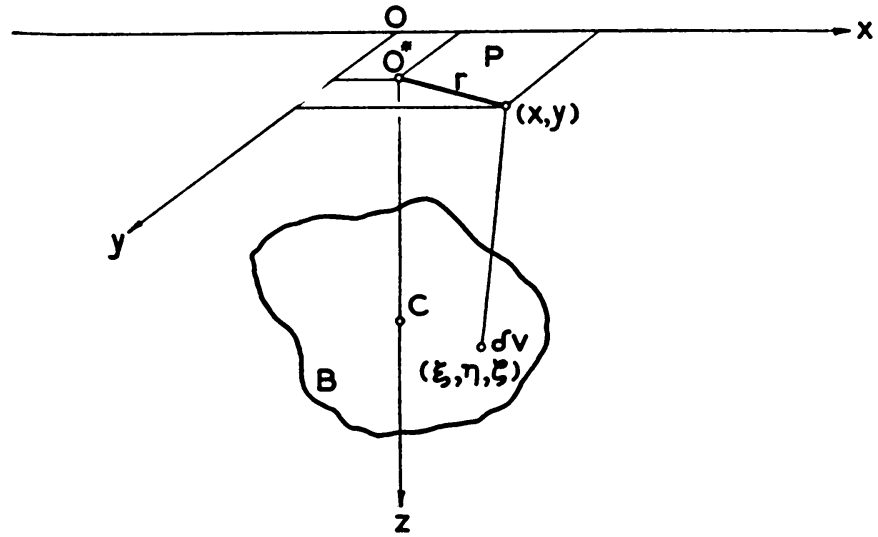

FIG. 2.

If $\delta V$ is an element of volume of $B$ at a point $(\xi, \eta, \zeta)$, and if $\delta[D g(x, y)]$ is the contribution to $D g$ from $\delta V$, then $\delta[D g]=f \sigma \zeta \delta V\left[(x-\xi)^{2}+(y-\eta)^{2}+\zeta^{2}\right]^{-3 / 2}$. Now $\iint_{P} \delta[D g] d S=2 \pi f \sigma \delta V$, which is independent of $\xi, \eta, \zeta$. Integration of this over $B$ yields $\iint_{P} D g d S=\pi k V$, where $k=2 f \sigma$ and $V$ is the volume of $B$. This result holds for any homogeneous irregular body or bodies.

Because of symmetry $\iint_{P}[x-\xi+i(y-\eta)] \delta[D g(x, y)] d S=0$. Thus

$$
\iint_{P}(x+i y) \delta[D g] d S=(\xi+i \eta) \iint_{P} \delta[D g] d S=\pi k(\xi+i \eta) \delta V,
$$

and integration over the body $B$ yields

$$
x^{*} \iint_{P} D g d S=\iint_{P} x D g d S, \quad y^{*} \iint_{P} D g d S=\iint y D g d S .
$$

These are two equations for $x^{*}$ and $y^{*}$. They hold for complex anomalies as well as simple ones. In practice, integration can be carried out only over a finite part of the plane $P$. We choose that part lying inside a circle with center $O$ and radius $R$, where $R$ is a constant at least four or five times the depth $z^{*}$ of $C$. The equations of this circle are $r=R, z=0$, where $r^{2}=x^{2}+y^{2}$. We denote its interior by $L$. The contribution to the above integrals from the infinite region $r \geqq R$ can be easily evaluated, since at such large distances the gravitational action of the body is approximately the same as that of a punctual mass $\sigma V$ located at the point $C\left(x^{*}, y^{*}, z^{*}\right)$. Therefore, for $r \geqq R$ we use the approximate formula

$$
\begin{aligned}
D g=f \sigma z^{*} V r^{-3}\{1+ & 3\left(x^{*} \cos \theta+y^{*} \sin \theta\right) r^{-1}+\left(9 x^{* 2}+9 y^{* 2}-6 z^{* 2}\right)(2 r)^{-2} \\
& \left.+15\left[2 x^{*} y^{*} \sin 2 \theta+\left(x^{* 2}-y^{* 2}\right) \cos 2 \theta\right](2 r)^{-2}+0\left(r^{-3}\right)\right\},
\end{aligned}
$$

where $r, \theta$ are polar coordinates in the plane $P$, with origin at $O$. Neglecting terms of order $\left(z^{*} / R\right)^{-3}$ and higher, we have with the aid of (19), 


$$
\begin{aligned}
\left(1-\frac{z^{*}}{R}\right) \iint_{P} D g d S & =\iint_{L} D g d S, \\
\left(1-\frac{3 z^{*}}{2 R}\right) \iint_{P}(x+i y) D g d S & =\iint_{L}(x+i y) D g d S .
\end{aligned}
$$

Thus (18) can be written in the form

$$
\left(x^{*}+i y^{*}\right) \iint_{L} D g d S=\left(1+\frac{z^{*}}{2 R}+\frac{3 z^{* 2}}{4 R^{2}}\right) \iint_{L}(x+i y) D g d S .
$$

Equations (20), applied in the first approximation with $z^{*}=0$, give a first position $O_{1}$ for $O^{*}$. If we choose the origin $O$ at this point, then $d=O_{1} O^{*}$ is small.

To obtain an equation for $z^{*}$, we integrate $r^{2} D g d S$ over $L$. Neglecting terms of relative order $\left(z^{*} / R\right)^{2}$ and higher, we need only the two first principal terms of this integral. Thus we can evaluate the contribution of an elemental volume $\delta V$ at $(\xi, \eta, \zeta)$ by integrating $r^{2} \delta[D g] d S$ over the region $r^{\prime} \leqq R$ instead of $L$, the origin of polar coordinates $\left(r^{\prime}, \theta^{\prime}\right)$ being at the point $(\xi, \eta)$ above the point $(\xi, \eta, \zeta)$. In fact, the difference between two integrals over $L$ and $r^{\prime} \leqq R$ is of relative order $\left(z^{*} / R\right)^{2}$. Now $r^{2}=r^{\prime 2}+\rho^{2}-2 \rho r^{\prime} \cos \left(\theta-\theta^{\prime}\right)$, and integration over $r^{\prime} \leqq R$ gives

$$
\iint_{L} r^{2} \delta[D g] d S=\pi k R \delta V\left\{\zeta+\left(\rho^{2}-2 \zeta^{2}\right) R^{-1}+\zeta 0\left(z^{* 2} / R^{2}\right)\right\} .
$$

Integrating this result over $V$ and replacing the integral of the second term by its approximate value $-2 k \pi z^{* 2} V$, we obtain

$$
\iint_{L} r^{2} D g d S=\pi k V z^{*} \dot{R}\left\{1-2 z^{*} R^{-1}+0\left(z^{* 2} / R^{2}\right)\right\} .
$$

Dividing by $R \iint_{L} D g d S=\pi k V R\left(1-z^{*} R^{-1}\right)$, we find that

$$
z^{*} R \iint_{L} D g d S=\left(1+z^{*} R^{-1}\right) \iint_{L} r^{2} D g d S .
$$

The term $\frac{1}{2} z^{* 2} R^{-2}$ must be added to the factor $1+z^{*} R^{-1}$ if the terms neglected are of order $\left(z^{*} / R\right)^{3}$ and higher.

When the measurements are performed with the aid of a gradiometer or torsion balance, the resulting maps of $U_{x z}$ and $U_{y z}$ give not only $x^{*}, y^{*}, z^{*}$ but also a control, since each of these two maps can be used to locate the point $C$. Applying the same reasoning as for $D g$, i.e., first integrating $\delta U_{x z}$ and $\delta U_{y z}$ corresponding to an elemental volume $\delta V$, and then integrating the result over $V$, we find that

$$
\begin{array}{ll}
x^{*} \iint_{L} x U_{x z} d S=\frac{1}{2} \iint_{L} x^{2} U_{x z} d S, & x^{*} \iint_{L} y U_{y z} d S=\iint_{L} x y U_{y z} d S, \\
y^{*} \iint_{L} x U_{x z} d S=\iint_{L} x y U_{x z} d S, & y^{*} \iint_{L} y U_{y z} d S=\frac{1}{2} \iint_{L} y^{2} u_{y z} d S .
\end{array}
$$

All the integrals in (22) have the same reduction factor $1+3 z^{*} / 2 R$. Hence it does not appear. The third reduced moments give equations for $z^{*}$. They are 


$$
\begin{aligned}
& 9 R z^{*} \iint_{L} x U_{x z} d S=8\left(1+\frac{7 z^{*}}{6 R}\right) \iint_{L} x^{3} U_{x z} d S=24\left(1+\frac{7 z^{*}}{6 R}\right) \iint_{L} x y^{2} U_{x z} d S, \\
& 9 R z^{*} \iint_{L} y U_{y z} d S=8\left(1+\frac{7 z^{*}}{6 R}\right) \iint_{L} y^{3} U_{y z} d S=24\left(1+\frac{7 z^{*}}{6 R}\right) \iint_{L} x^{2} y U_{y z} d S .
\end{aligned}
$$

If we neglect only terms of order $\left(z^{*} / R\right)^{3}$ and higher, the term $11 z^{* 2} /\left(18 R^{2}\right)$ must be added to the reduction factor $1+7 z^{*}(6 R)^{-1}$. Equations (22) and (23) are general. For a solid of revolution with a vertical axis, they reduce to

$$
\begin{aligned}
\left(x^{*}+i y^{*}\right) \iint_{L} G d S & =\left(1+\frac{3 c z^{*}}{4 R}\right) \iint_{L}(x+i y) G d S, \\
3 R z^{*} \iint_{L} r G d S & =2\left(1+\frac{7 z^{*}}{6 R}+\frac{11 z^{* 2}}{18 R^{2}}\right) \iint_{L} r^{3} G d S,
\end{aligned}
$$

where $c$ is such that $R c \iint_{L} G d S=\iint_{L} r G d S$.

In the general case of a complex magnetic anomaly we assume that the magnetization vector is the same for all particular isolated bodies which create this anomaly. Its intensity $I$, inclination $\psi$, and azimuth $\phi$ are three unknowns; $I$ cannot be separated from the total volume $V$ and it is the product $V I$ which is deduced from the maps; $\phi$ is defined with respect to arbitrary cartesian axes of $x$ and $y$ on the surface of the earth; $X, Y$ and $Z$ are the components of the anomaly.

We shall now deduce expressions for the six parameters $V I, \psi, \phi, x^{*}, y^{*}, z^{*}$ which characterize the magnetization vector and locate the common center of gravity of all the disturbing magnetic bodies. This can be done by computing the moments of $X, Y, Z$ from maps showing the distribution of $X, Y, Z$ over the surface of the earth, and by use of the classical Eötvös formulae,

$$
\begin{aligned}
f_{\sigma}(\mathrm{i} X+\mathrm{j} Y & +\mathrm{k} Z) \\
& =I\left(\mathrm{i} \frac{\partial}{\partial x}+\mathrm{j} \frac{\partial}{\partial y}+\mathrm{k} \frac{\partial}{\partial z}\right)\left[\left(U_{x} \cos \phi+U_{y} \sin \phi\right) \cos \psi+U_{z} \sin \psi\right],
\end{aligned}
$$

where $i, j, k$ are unit vectors on the coordinate axes. We shall use moments of the first four orders, denoting them by subscripts. For example, $X_{m n}=\iint_{L} X x^{m} y^{n} d S$. It is to be noted that $X_{m n}$ are reduced moments. Neglecting terms of relative order $\left(z^{*} / R\right)^{2}$, and using the same method as in the case of (21) in the computation of integrals of the second derivatives of $U$, we obtain

$$
-X_{00} \sec \psi \sec \phi=-Y_{00} \sec \psi \csc \phi=2 Z_{00} \csc \psi=2 \pi V I / R \text {. }
$$

The first approximate values of $V I, \psi, \phi$ are then given by

$$
\tan \phi=Y_{00} / X_{00}, \tan \psi=\frac{1}{2} Z_{00}\left(X_{00}^{2}+Y_{00}^{2}\right)^{-1 / 2}, \pi V I=R\left(X_{00}^{2}+Y_{00}^{2}+\frac{1}{4} Z_{00}^{2}\right)^{1 / 2} \text {. }
$$

The zero moments are small quantities, and approach zero as $R$ approaches infinity. Hence (24) yield poor approximations. Nevertheless, the first value of $\phi$ must be used to rotate the axes of coordinates, directing the $x$-axis nearly parallel to the horizontal component of the magnetization vector, so that $\phi$ will be very small.

Among the six moments of the first order, we do not use $X_{01}$ and $Y_{10}$ since they are of order $1 / R$ compared with the other four, which are given by 


$$
\begin{aligned}
\frac{X_{10}}{2 \pi V I} & =-\sin \psi\left(1+\frac{x^{*}}{2 R} \cot \psi \cos \phi\right), & \frac{Y_{01}}{2 \pi V I} & =-\sin \psi\left(1+\frac{y^{*}}{2 R} \cot \psi \sin \phi\right), \\
\frac{Z_{10}}{2 \pi V I} & =-\cos \psi\left(\cos \phi-\frac{x^{*}}{R} \tan \psi\right), & \frac{Z_{01}}{2 \pi V I} & =-\cos \psi\left(\sin \phi-\frac{y^{*}}{R} \tan \psi\right) .
\end{aligned}
$$

Hence we obtain better values for $V I$ and $\tan \psi$,

$$
\begin{aligned}
& 2 \pi V I=\left(Z_{10}^{2}+Z_{01}^{2}+\frac{1}{2} X_{10}^{2}+\frac{1}{2} Y_{01}^{2}\right)\left(1+\frac{c_{1}}{R}\right), \\
& \tan \psi=2^{-1 / 2}\left(X_{10}^{2}+Y_{01}^{2}\right)^{1 / 2}\left(Z_{10}^{2}+Z_{01}^{2}\right)^{-1 / 2}\left(1-\frac{c_{2}}{R}\right),
\end{aligned}
$$

where

$$
\begin{gathered}
8 c_{1}=3 \sin 2 \psi\left(x^{*} \cos \phi+y^{*} \sin \phi\right)=6 \cos ^{2} \psi \cdot c_{3} \\
4 c_{2}=(\cot \psi+4 \tan \psi)\left(x^{*} \cos \phi+y^{*} \sin \phi\right)=\left(4+\operatorname{cotan}{ }^{2} \psi\right) c_{3} \\
c_{3}=2^{-3 / 2}\left(X_{10}^{2}+Y_{10}^{2}\right)^{1 / 2}\left(Z_{01}^{2}+Z_{10}^{2}\right)^{-2}\left[\left(Z_{20}-Z_{02}\right)\left(Z_{01}^{2}-Z_{10}^{2}\right)-4 Z_{01}^{2} Z_{11} Z_{10}\right] .
\end{gathered}
$$

The coordinates $x^{*}, y^{*}$ of the point $O^{*}$ are obtained with the aid of second moments, as indicated by the equations

$$
\begin{aligned}
& x^{*}=\frac{1}{2}\left(Z_{10}^{2}+Z_{01}^{2}\right)^{-1}\left[\left(Z_{20}-Z_{02}\right) Z_{10}+2 Z_{01} Z_{11}\right]\left(1-\frac{c_{3}}{R}\right)+\frac{y^{* 2}}{R} \tan \psi \cos \phi, \\
& y^{*}=\frac{1}{2}\left(Z_{10}^{2}+Z_{01}^{2}\right)^{-1}\left[2 Z_{10} Z_{11}-\left(Z_{20}-Z_{02}\right) Z_{01}\right]\left(1-\frac{c_{3}}{R}\right)+\frac{x^{* 2}}{R} \tan \psi \sin \phi .
\end{aligned}
$$

The expression

$$
Y_{20}=\frac{5}{2} R \cos \psi \sin \phi\left(1-\frac{12 z^{*}}{5 R}\right)
$$

shows that $Y_{20}$ is zero if the $x$-axis is parallel to the horizontal component of the magnetization vector $I$. If $Y_{20}$ is different from zero, the value of $\phi$ is given with good precision by the important equation $X_{02}=Y_{20} \tan \phi$. Thus, by use of this equation and Eqs. (25), we can change our axes so that $x^{*}=y^{*}=\phi=0$. Computing the third moments in this new system of coordinates, we find that $X_{21}=X_{03}=Y_{30}=Y_{12}=Z_{21}=Z_{03}$ $=0,3 Z_{12}=Z_{30}=X_{30} \cot \psi$,

$$
3 X_{12}=3 Y_{21}=X_{30}=Y_{03}=-\frac{9}{8} R z^{*}\left(1-\frac{7 z^{*}}{6 R}\right) \sin \psi \text {. }
$$

These expressions give for $\tan \psi$ the eight values $X_{30} / Z_{30}, Y_{21} / Z_{12}$, etc., and the mean of these eight values can be considered as the final value of $\tan \psi$. Now, with this choice of axes, the second moments take the values $X_{11}=Y_{20}=Y_{02}=Z_{11}=0$,

$$
\begin{array}{ll}
X_{20}=\frac{5}{2} R\left(1-\frac{16 z^{*}}{5 R}\right) \cos \psi, & X_{02}=\frac{5}{2} R\left(1-\frac{12 z^{*}}{5 R}\right) \cos \psi, \\
Y_{11}=\frac{3}{8} R\left(1-\frac{8 z^{*}}{3 R}\right) \cos \psi, & Z_{20}=Z_{02}=-5 R\left(1-\frac{14 z^{*}}{5 R}\right) \sin \psi .
\end{array}
$$


Dividing any third moment by one of the five second moments different from zero, we obtain an approximate value for $z^{*}$, and the mean of all such values can be considered as the final value of $z^{*}$. Thus, we have not only solved the problem, but have also found a control of the solution, since the degree of concordance of many different values found for the same parameter, such as $z^{*}$, characterizes the reliability of the solution.

Fom a practical point of view, a solution involving only the moments of the vertical component $Z$ is important, since in many cases magnetic surveys are limited to measurements of $Z$ only. In such cases we can find $\psi$ and $\phi$ and locate the point $O^{*}$ by use of Eqs. (25) together with

$$
\begin{aligned}
Z_{10} \tan \phi & =Z_{01}\left(1+\frac{y^{*}-x^{*}}{R} \tan \psi\right), \\
5 R\left(Z_{10}^{2}+Z_{01}^{2}\right)^{1 / 2} \tan \psi & =\left(1+\frac{c_{4}}{R}\right)\left[\left(Z_{20}-2 x^{*} Z_{10}\right)^{2}+\left(Z_{02}-2 y^{*} Z_{01}\right)^{2}\right]^{1 / 2},
\end{aligned}
$$

where $c_{4}$ is such that $5 R c_{4}=14 z^{*}-\left(x^{*} \cos \phi+y^{*} \sin \phi\right) \tan \psi$. For VI we have

Also, $z^{*}$ is given by

$$
10 \pi R V I=\left(1+\frac{c_{4}}{R}\right)\left(Z_{20}^{2}+Z_{02}^{2}\right)^{1 / 2} .
$$

$$
z^{*}=-\frac{8}{9}\left(1+\frac{7 z^{*}}{6 R}\right) \frac{Z_{30}}{R}\left(Z_{10}^{2}+Z_{01}^{2}\right)^{1 / 2},
$$

where the moments are related to the origin $O^{*}$ with $\phi=0$. It is plain that the accuracy of the approximate values given by the above equations increases with $R$.

4. Interpretation of the map of an anticline with the aid of moments. Let us represent the structure of an anticline as a cylindrical body of normal cross section $S$. We have first to choose for $S$ some simple geometrical form which expresses the gravitational action of the anticline adequately. Our choice is the region between two concentric elliptic cylinders, the major axes of which have an angle $\alpha$ of inclination (Fig. 3). This choice is good, except in the vicinity of the deepest part of the region, which part is at a considerable depth; the author has verified that such a choice leads to good results in practise. We shall denote the outer and inner ellipses by $E_{1}$ and $E_{\gamma}$, respectively, and their semi-axes by $a, b$ and $\gamma a, \gamma b$, respectively, where $\gamma$ is a positive constant less than unity.

Equations (10)-(14) give values for $k A, x^{*}, z^{*}$. We shall now show how $\gamma, \alpha$ and the eccentricity $e$ can be deduced from graviţaticnal anomaly maps.

Let us replace $e$ by a parameter $r=2 z^{*} / c$, where $2 c$ is the focal distance of the outer ellipse. We define a function $n(s)$ by the relation

$$
n(s)=M_{0}^{2 s-1} M(-s) D^{-s},
$$

where $s$ is a constant and $0<s<1, M_{0}$ is as defined in $\S 3, M(-s)$ is the moment of $D g$ of order $-s$, and $D$ is the zero moment of $[D g(x)]^{2}$ :

$$
M(-s)=\int_{-\infty}^{\infty}|x|^{-s} D g d x, \quad D=\int_{-\infty}^{\infty}[D g(x)]^{2} d x .
$$


The graphs of $|x|^{-s} D g(x)$ and $[D g(x)]^{2}$ can be plotted from the experimental curve of $D g(x)$, and thus the reduced moments can be computed with the aid of a planimeter. On the other hand $n(s)$ can be tabulated for various values of $s$. We shall require $n\left(\frac{1}{4}\right), n\left(\frac{1}{2}\right), n\left(\frac{3}{4}\right)$.

Applying the theory of Fourier transforms to (16), (17), we have

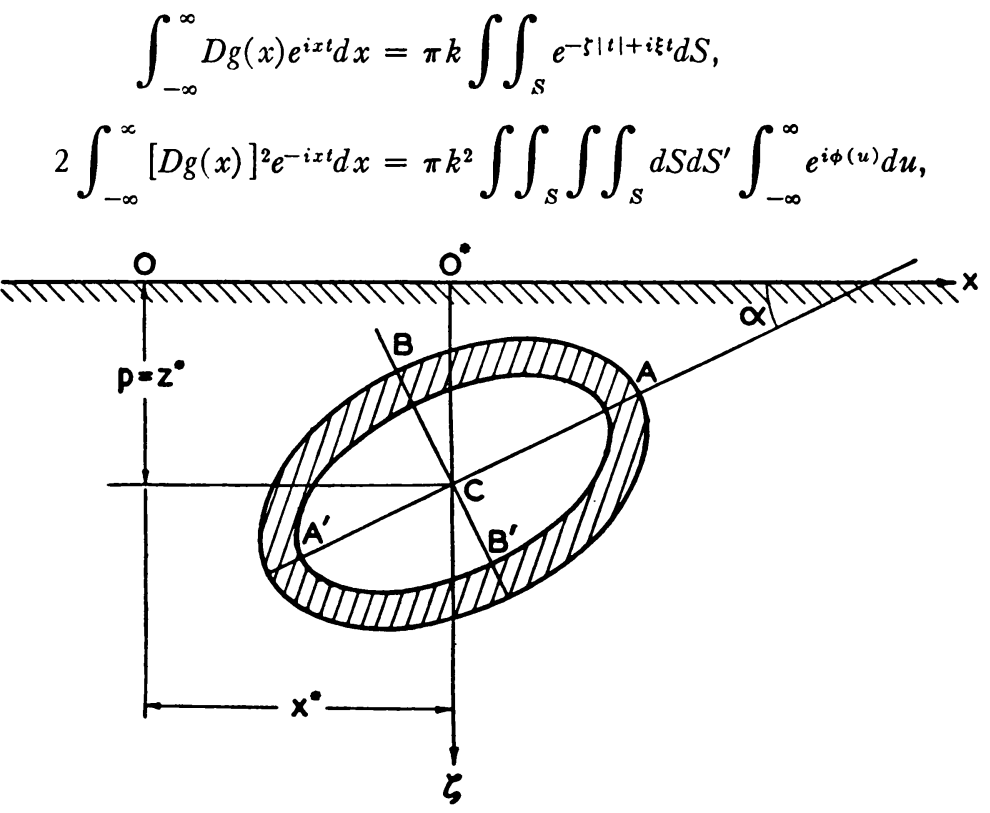

FIG. 3.

where $\phi(u)=\xi t+\left(\xi^{\prime}-\xi\right) u-\zeta^{\prime}|u|-\zeta|t-u|$, and $(\xi, \zeta),\left(\xi^{\prime}, \zeta^{\prime}\right)$ are two sets of running coordinates on $S$. We note that

$$
\int_{t}^{\infty} e^{i v y}(y-t)^{-\delta} d y=(i v)^{\delta-1} e^{i v t} \Gamma(1-\delta)
$$

for $t \geqq 0,0<\delta<1, \operatorname{Re}[i v] \leqq 0$, where $\operatorname{Re}[i v]$ denotes the real part of $i v$. Consequently, the application to (27) of fractional integration of order $1-\delta$ yields for $t \geqq 0$,

$$
\int_{-\infty}^{\infty} D g(x)|x|^{\delta-1} e^{i x t} d x=\pi k \iint_{S} \rho^{\delta-1} e^{i \rho t} d S .
$$

The inversion of order of integration is permissible, since all integrals are absolutely convergent. Replacing in (29) the exponent $\delta-1$ by $-s,(0<s<1)$, multiplying both sides of this by $e^{i \pi s / 2}$ and letting $t$ tend to zero, we obtain

$$
M(-s)=\int_{-\infty}^{\infty}|x|^{-8} D g(x) d x=\pi k \sec \left(\frac{1}{2} \pi s\right) \operatorname{Re}[\Phi(A ;-s)],
$$

where $\Phi(A ;-s)=e^{i \pi s / 2} \iint_{\mathcal{S}} \rho^{-8} d S$. For our region $S$ we have 


$$
\begin{aligned}
\Phi(A ;-s)=A\left(z^{*}\right)^{-s}\left[F \left(\frac{1}{2} s, \frac{1}{2}+\frac{1}{2} s, 2 ;-\right.\right. & \left.4 r^{-2} e^{-2 i \alpha}\right) \\
& \left.\quad-\gamma^{2} F\left(\frac{1}{2} s, \frac{1}{2}+\frac{1}{2} s, 2 ;-4 r^{-2} \gamma^{2} e^{-2 i \alpha}\right)\right],
\end{aligned}
$$

where $F(a, b, c ; x)$ is the classical hypergeometric function. The function $M(-s)$, which depends on the four parameters $s, \alpha, \gamma, r$, can be tabulated for $-1<s<1$, $0 \leqq \gamma \leqq 1,0 \leqq \alpha \leqq \frac{1}{2} \pi, r \geqq 2 \sin \alpha$. We shall require tabulations for $s=\frac{1}{4}, \frac{1}{2}, \frac{3}{4}$.

If in the right side of (28) we carry out the integration with respect to $u$ for $t \geqq 0$, and then let $t$ approach zero, we find that

$$
2 D=2 \int_{-\infty}^{\infty}[D g(x)]^{2} d x=i \pi k^{2} \iint_{S} \iint_{S}\left(\rho-\bar{\rho}^{\prime}\right)^{-1} d S d S^{\prime},
$$

where $\rho=\xi+i \zeta, \bar{\rho}^{\prime}=\xi^{\prime}-i \zeta^{\prime}$. In the present case, we have

$$
D=M_{0}^{2}\left(z^{*}\right)^{-1} \phi(\alpha, \gamma ; r),
$$

where

$$
\begin{aligned}
& \phi(\alpha, \gamma ; r)=f\left(e^{-i \alpha}, e^{i \alpha} ; r\right)-2 R e\left[f\left(\gamma e^{-i \alpha}, e^{i \alpha} ; r\right)\right]+f\left(\gamma e^{-i \alpha}, \gamma e^{i \alpha} ; r\right), \\
& 3 f(u, v ; r)=16 r u^{3} v^{3}(u+v)^{-2}\left[C_{1} E-C_{2} K+C_{3} K E\left(b, \lambda^{\prime}\right)\right],
\end{aligned}
$$

$K$ and $E$ being complete elliptic integrals of the first and second kind of modulus $\lambda$ given by $4 u v=\lambda^{2}\left[r^{2}+(u+v)^{2}\right], \lambda^{\prime}$ and $b$ being such that $\lambda^{\prime}=\left(1-\lambda^{2}\right)^{1 / 2}, 2(u v)^{1 / 2} d n\left(b, \lambda^{\prime}\right)$ $=\lambda \cdot(u+v)$, and $C_{1}, C_{2}, C_{3}$ such that

$$
\begin{aligned}
& C_{3}=3 \lambda^{\prime}\left[1-\lambda^{\prime} \operatorname{sn}\left(b, \lambda^{\prime}\right) c n\left(b, \lambda^{\prime}\right) d n\left(b, \lambda^{\prime}\right)\right], \\
& C_{1}=3 d n^{2}\left(b, \lambda^{\prime}\right)+3 \lambda^{\prime} s n\left(b, \lambda^{\prime}\right)-1-\lambda^{2}+b C_{3}, \\
& C_{2}=3 \lambda^{\prime 2} d n^{2}\left(b, \lambda^{\prime}\right) c n^{2}\left(b, \lambda^{\prime}\right)+3 \lambda^{\prime} s n\left(b, \lambda^{\prime}\right)\left[1+\lambda^{\prime 2} c n^{2}\left(b, \lambda^{\prime}\right)\right]+\lambda^{\prime 2}+b C_{3} .
\end{aligned}
$$

Thus $\phi(\alpha, \gamma ; r)$ can be tabulated.

We have now derived expressions for $M(-s)$ and $D$ occurring in the right side of (26). Substitution yields the equation

$$
E(s ; \alpha, \gamma ; r)=n(s)
$$

where $E(s ; \alpha, \gamma ; \gamma)=[\phi(\alpha, \gamma ; r)]^{-s} m(-s ; \alpha, \gamma ; r)$, with

$$
m(-s ; \alpha, \gamma ; r)=\sec \left(\frac{1}{2} \pi s\right) A^{-1} z^{* s} \operatorname{Re}[\Phi(A ;-s)] .
$$

In (30) we set $s=\frac{1}{4}, \frac{1}{2}, \frac{3}{4}$, to obtain three equations which we can solve for the required quantities $\alpha, \gamma, r$.

Once $\alpha, \gamma, r$ have been determined, we can deduce a new value for $z^{*}$ from the relation

$$
z^{*}=M_{0}^{2} D^{-1} \phi(\alpha, \gamma ; r)
$$

This serves as a control on the value obtained from (12).

In actual computations based on experimental data, we can use only a finite region on the surface of the earth. Hence reduced moments must be introduced, as before, and the parameters of the problem must be deduced by successive approximations.

The same procedure can be used in treating maps of the gradient $G$ and the curva- 
ture $K$. Each map leads to an independent set of values for $x^{*}, z^{*}, \alpha, \gamma, r, k A$. In the case of a magnetic anomaly, the map of $X$ or of $Z$ could be used. In all cases the moments can be expressed in terms of $\Phi(A ;-s)$.

We note that the above method yields $k A$. Now $A$ is the area of the cross section and $k=2 \sigma$, where $f$ is the gravitational constant and $\sigma$ is the density-contrast. We define the average thickness $T$ of the disturbing layer as the geometric mean of the extreme thicknesses; $T=(1-\gamma)(a b)^{1 / 2}$. Since $A=\pi a b\left(1-\gamma^{2}\right)$, we then have $A(1-\gamma)$ $=\pi(1+\gamma) T^{2}$. Hence, if $\sigma$ is known, $A$ can be found and then $T$. Often $T$ is known. In this case, $A$ can be found, and then $\sigma$.

5. Interpretation of a centered anomaly created by a salt dome. Before applying the new method it is interesting to see what can be obtained by the old method. Some results can be achieved if we disregard the cap-rock, neglect the slope of the flanks and omit the depth of the salt dome base considering this structure as a homogeneous vertical infinite circular cylinder whose top is at the depth $p$ (Fig. 4). The interpretation problem is reduced to finding the three parameters: $k(\sigma=k / 2 f), p$ and the radius $a$ of the dome. Instead of $a$ we consider the angle $\omega$, letting $a=p \tan \omega$. With the origin at $O^{*}$, just above the center of the top circle (maximum of $D g$, zero for the gradient $G$ ) the expressions for $G$ and $D g$ are:

$$
\begin{aligned}
G(r) & =k(a / r)^{1 / 2}\left[2 \lambda^{-1}(K-E)-\lambda K\right], \\
D g(r) & =k\left[C_{1} E+C_{2} K-\pi p f(r)\right],
\end{aligned}
$$

where $C_{1}=b p \operatorname{sign}(a-r)+2(a r)^{1 / 2} \lambda^{-1}$,

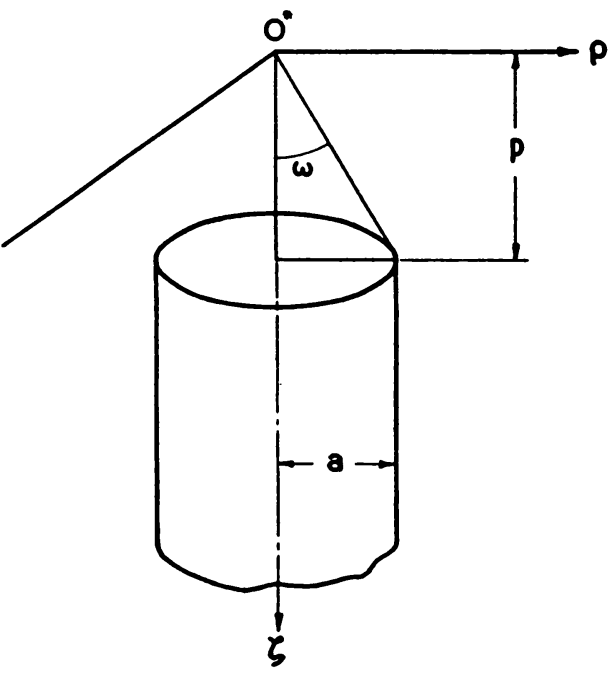

Fig. 4. $C_{2}=\lambda\left(a^{2}-r^{2}\right)(4 a r)^{-1 / 2}+p\left[E\left(b, \lambda^{\prime}\right)-b\right] \operatorname{sign}(a-r)$ and $2 f(r)=1+\operatorname{sign}(a-r)$, sign 0 being defined by sign $0=0$. The elliptic function $E\left(b, \lambda^{\prime}\right)$ and the complete integrals $K$, $E$ have the moduli $\lambda^{\prime}=\left(1-\lambda^{2}\right)^{1 / 2}$ and $\lambda$ is defined by $\lambda\left[p^{2}+(a+r)^{2}\right]^{1 / 2}=2(a r)^{1 / 2}$, $r$ being the distance from the origin. The argument $b\left(0 \leqq b \leqq K^{\prime}\right)$ is that in $d n\left(b, \lambda^{\prime}\right) \cdot\left[p^{2}+(a+r)^{2}\right]^{1 / 2}=a+r$. Either of two curves $G=G(r), D g=D g(r)$ can be deduced from the other by graphical differentiation or integration. Therefore, we can use both of them for the interpretation.

Here it is the maximum of the product $F(r)=r^{1 / 2}|G(r)|$ which it is important to locate and therefore the curve $G$ must be transformed into the graph of the function $F(r)$. In fact, the equation $F^{\prime}(r)=0$ reduces to $\lambda^{3} E\left(p^{2}+a^{2}-r^{2}\right)=0$ and the maximum of $F(r)$ corresponds to $r=r_{0}=\left(p^{2}+a^{2}\right)^{1 / 2}$. The corresponding value of the modulus $\lambda$ is $\lambda_{0}=\left[1-\tan ^{2}(\pi / 4-\omega / 2)\right]^{1 / 2}$. The value of the maximum itself is $\max F(r)=F_{0}=k(p \tan \omega)^{1 / 2}\left[2 \lambda_{0}^{-1}\left(E_{0}-K_{0}\right)+\lambda_{0} K_{0}\right]$. Thus, we have at our disposal three experimental data $r_{0}, F_{0}$ and the maximum $D g_{0}=\pi k p(\sec \omega-1)$ of $D g(r)$. First we find the angle $\omega$, solving the equation $B(\omega)=n$, where the function $B(\omega)$ is defined by $\pi \sin (\omega / 2)(1+\sin \omega-\cos \omega) B(\omega)=-2^{1 / 2}\left[(1+\sin \omega) E_{0}-K_{0}\right]$, the modulus $\lambda_{0}$ being a function of $\omega$ only. The value of the number $n$ is deduced from the measure- 
ments by the rule $n=r_{0}^{1 / 2} F_{0} / D g_{0}$, this value being simply the ratio of two maxima, multiplied by the square root of the observed distance $r_{0}$. The function $B(\omega)$ is easily tabulated. Knowing $\omega$, we have $p=r_{0} \cos \omega$ and $a=p \tan \omega$. The value of $k$ is deduced from $F_{0}$ or $D g_{0}$. That is all that can be obtained, using the old method, and it is evident that it cannot satisfy a geologist. In practice the best value for $r_{0}$ is $(S / \pi)^{1 / 2}$, where $S$ denotes the area of the closed curve, the locus of maxima of $F(r)$ on all radial profiles through the origin $O^{*}$. This rather rough method of interpretation works if it is applied to an isolated salt dome. The advantage of the inaccurate first approximation which it gives consists in the simplicity of computations. The interpretation requires only the table of values of the function $B(\omega)$ and it can be performed as fieldwork and very rapidly.

We shall now apply the new method. It is assumed that the salt dome is a solid of revolution. Cartesian axes are chosen, with the $z$-axis directed downward along the axis of revolution, and the origin $O^{*}$ on the surface of the earth. We denote the cylindrical coordinates of a general point inside the solid of revolution by $(\rho, \phi, \zeta)$, and of a general point outside by $(r, \theta, z)\left(z<\zeta_{\min }\right) ; R$ is the distance between these two points. By means of the classical formula

$$
\int_{0}^{2 \alpha} \frac{d \phi}{R}=2 \pi \int_{0}^{\infty} e^{-(\zeta-z) u} J_{0}(r u) J_{0}(\rho u) d u,
$$

where $J_{\mathrm{n}}(t)$ is a Bessel function, we deduce for the potential

where

$$
U(r, z)=\int_{0}^{\infty} e^{u z} W(u) J_{0}(r u) d u,
$$

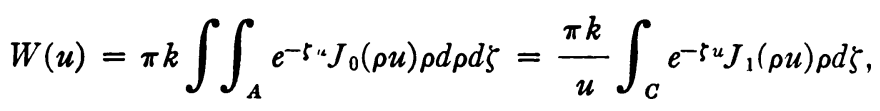

$A$ being the region the revolution of which generates the solid, and $C$ being its boundary. Denoting the Hankel transform of order $r$ by $H_{r}$, so that by definition

$$
F(r)=H_{r}[f(u)]=\int_{0}^{\infty} f(u) J_{r}(r u) u d u,
$$

for $z=0$ we have $U(r, 0)=H_{0}[W(u) / u]$. Differentiation of (31) yields similar expressions for $U_{z}=D g(r), U_{z z}, G, K$ :

$D g(r)=H_{0}[W(u)], \quad C_{z z}=H_{0}[u W(u)], \quad-G=H_{1}[u W(u)], \quad K=H_{2}[u W(u)]$.

The advantage presented by the expressions (32) consists in the possibility of using the theorems of the transform theory in calculating the moments and moment functions used in the interpretation. Because of lack of space we can give as example only the general expression for the moment function of $D g$ which holds for any form of the solid of revolution, the expression applied below to the interpretation of the gravity map of a salt dome. There is another approach to the mathematical problem of computing the moments and moment functions needed in the interpretation. Indeed, direct integration of the explicit formulae (32) is easily performed with the aid 
of divergent and summable integrals of Bessel functions of the general type (with $a$ and $b$ restricted only by $a+b>-1$ ),

$$
\int_{0}^{\infty} t^{a} J_{b}(u t) d t \cong 2^{a} u^{-(a+1)} \Gamma[(a+b+1) / 2]\{\Gamma[(b-a+1) / 2]\}^{-1},
$$

but we prefer to use the transform theory. A salt dome creates also a magnetic anomaly and, for a solid of revolution, $\left(15^{*}\right)$ gives the expressions for its components on the ground $z=0$ with the aid of gravitational quantities $G, K$ and $U_{z z}$. Using the results of Section 3, we choose the origin and the $x$-axis so that $x^{*}=y^{*}=0, \phi=0$. Under this assumption

$$
\begin{aligned}
k(X+i Y) & =I\left[\cos \psi\left(K e^{2 i \theta}-U_{z z}\right)+2 G \sin \psi e^{i \theta}\right], \\
k Z & =2 I\left(\sin \psi U_{z z}+G \cos \psi \cos \theta\right) .
\end{aligned}
$$

Instead of $X, Y$ it is convenient to use in the interpretation the radial and transversal components $A_{r}$ and $A_{t}$ of the horizontal anomaly $\left(X^{2}+Y^{2}\right)^{1 / 2}$. Transforming the $X$ and $Y$-maps into the maps of $A_{r}$ and $A_{t}$, we can consider their moments and moment functions as experimental data. The corresponding expressions in terms of parameters of the problem are deduced with the aid of

$$
k\left(A_{\mathbf{r}}+i A_{t}\right)=I\left[\cos \psi\left(K e^{i \theta}-U_{\mathbf{z} z} e^{-i \theta}\right)+2 G \sin \psi\right] .
$$

With the aid of (34), (35) all the rules for the interpretation of the gravitational anomaly can be adapted to the interpretation of magnetic anomalies produced by a solid of revolution. Far from the origin $O^{*}$ the gravitational action of a body can be approximately expressed as that of a material point of the same excess-mass $M=\sigma V$ located at the center of gravity $C\left(0,0, z^{*}\right)$. Thus, for large $r$ we have the approximate formulae

$$
D g \sim f M z^{*} r^{-3}, \quad-G \sim 3 f M z^{*} r^{-4}, \quad K \sim 3 f M r^{-3}, \quad U_{z z} \sim-f M r^{-3},
$$

the neglected terms being of the relative order $\left(z^{*} / r\right)^{2}$. For the magnetic quantities $A_{r}, Z$ we have, denoting the volume of the salt dome by $V_{s}$ (the caprock does not create a magnetic anomaly),

$A_{r} \sim I V_{\mathrm{g}} r^{-3}\left(2 \cos \psi \cos \theta-3 z^{*} r^{-1} \sin \psi\right),-Z \sim I V_{\mathrm{s}} r^{-3}\left(\sin \psi+3 z^{*} r^{-1} \cos \psi \cos \theta\right)$.

The moment functions are defined by the integrals extended over the infinite plane $P$ and the formulae (36), (37) are used in computing the reduction factors introduced by the integration over the finite area $r \leqq R$, denoted by $L$.

The formula (32) $D g(r)=H_{0} W(u)$ gives immediately $W(u)=H_{0} D g(r)$, that is,

$$
2 \pi W(u)=\iint_{P} D g(r) J_{0}(r u) d S .
$$

If the form of the solid is considered as known, only its dimensions being asked, i.e., if the expression of $W(u)$ as function of the parameters is prescribed, the relation (39) becomes the source of equations since for every particular value of the arbitrary parameter $u$ (which is the reciprocal of a length) it is an equation in the unknown parameters of the problem. In practice the numerical value of the second member is 
obtained by integrating over $L$, and adding to the result the contribution of the infinite area $r \geqq R$. This contribution, computed with the aid of (36), is equal to $2 \pi f M\left(z^{*} / R\right)\left[J_{1}(R u) / R u\right]$ where, as has been seen in Section $3,2 \pi f M=\pi k V$ is the value of the first moment of $D g$. In this method the map of $D g$ is transformed, for each value of $u$, into the map of the product $D g(r) J_{0}(r u)$ and the integration over $L$, giving the second member of (38), is performed on this map. Let us consider as an example the special case when the flanks of the dome are vertical. In this case the area $A$ is formed by two rectangles (see Fig. $5^{*}$ ) and there are seven parameters: the radii $a$ (salt) and $b$ (cap-rock) of two cylinders which together form the dome with its caprock, the depth $p$ at which the salt begins (bottom of the cap-rock), the thickness $h$

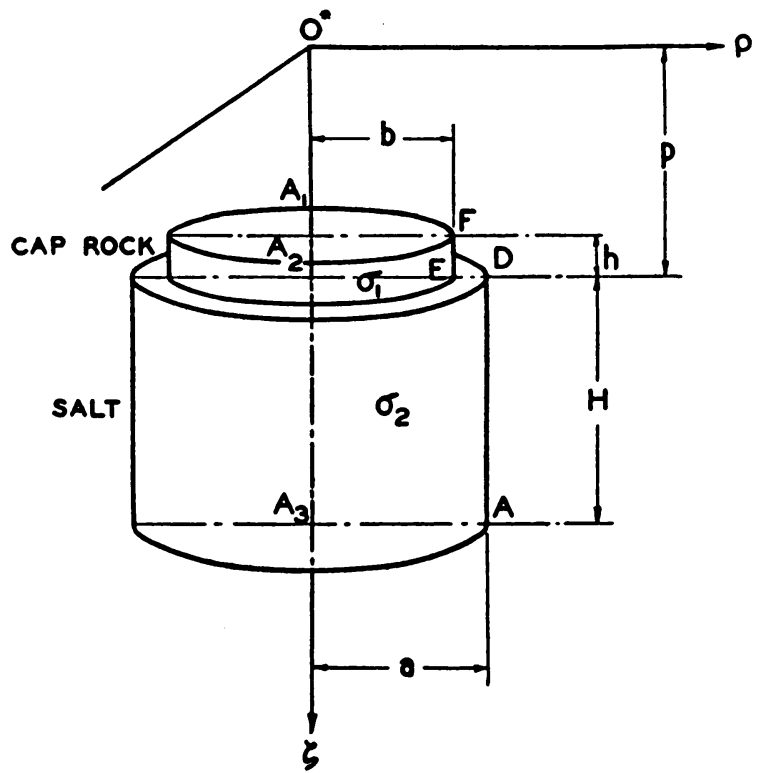

FIG. 5. of the cap-rock and that $H$ of the salt, the density-contrasts $k_{1}$ and $k_{2}$ (salt). The expression (31) gives the characteristic function $W(u)$,

$$
\begin{aligned}
& \pi^{-1} u^{2} W(u)=e^{-u p}\left[a k_{2} J_{1}(a u)\right. \\
& \left.\quad\left(1-e^{-u H}\right)+b k_{1} J_{1}(b u)\left(e^{u h}-1\right)\right] .
\end{aligned}
$$

Choosing any seven numerical values for $u$ and computing for them the corresponding values of the product $\pi^{-1} u^{2} W(u)$ with the aid of the rule (38), we have a system of seven equations with seven unknowns $a, b, k_{1}, k_{2}, p, H, h$. Solving it we have all the necessary informations about the dome. This method can be applied only if the flanks of the dome are vertical. Let us now consider the case of a deeply buried salt dome, substituting for it as an idealized form a truncated cone with the vertex at $O^{*}$. This assumption is not acceptable for shallow domes. We need the moment function and we begin by giving its general expression which holds for all solids of revolution. A. Erdelyi and H. Kober have recently proved $^{2}$ an important theorem on the Hankel transform which they formulate using Tricomi's form of this transform. In our notation (Hankel's form) this theorem states that if $W=H_{s+2 \alpha} w$, where $s>-1, \alpha>0$, and $u^{1 / 2} w(u)$ belongs to $L_{2}(0, \infty)$ then $T_{s \alpha}(W)=H_{s}\left[T_{s \alpha}(w)\right]$, the operator $T_{s \alpha}$ being defined by

$$
\Gamma(\alpha) T_{s \alpha} f(x)=2 x^{s} \int_{x}^{\infty}\left(y^{2}-x^{2}\right)^{\alpha-1} y^{1-s-2 \alpha} f(y) d y .
$$

Applying it to our relation $W(u)=H_{0} D g(r)$ with $-1<s<0, s+2 \alpha=0$, we have

$$
\int_{x}^{\infty}\left(y^{2}-x^{2}\right)^{-(1+s / 2)} W(y) y d y=x^{-s} \int_{0}^{\infty} J_{s}(x t) t^{t+1} d t \int_{t}^{\infty}\left(r^{2}-t^{2}\right)^{-(1+s / 2)} D g(r) r d r .
$$

* In Fig. 5 the letters $A_{1}$ and $A_{2}$ denote the centers of the two end surfaces of the cap rock cylinder.

${ }^{2}$ Quarterly Journal of Math., 2, 217 (1940), Theorem 5, case b. 
Interchanging the integrations in the second member (absolutely convergent double integral), using $t=r v^{1 / 2}$ and passing to the limit $x=0$, we get the desired general expression for the moment function $M(s)$ of $D g(r)$ for $-2<s<0$,

$$
\begin{aligned}
M(s) & =\iint_{P} D g(r) r^{s} d S=2 \pi \int_{0}^{\infty} D g(r) r^{s+1} d r \\
& =2^{2+8} \Gamma^{2}(1+s / 2) \sin (-s \pi / 2) \int_{0}^{\infty} W(u) u^{-(s+1)} d u .
\end{aligned}
$$

The same result can be obtained integrating the relation $r^{s+1} D g=H_{0} r^{s+1} W(u)$ under the sign of integration in $u$ and applying the integral (33). Now the integral in the second member of (40) can be calculated, using (31) and the formula (3) p. 385, ch. 13.2 of "Bessel Functions" by G. N. Watson, 1922. Let $\omega$ be the angle the radius vector of a point $(\rho, \zeta)$ makes with the $z$-axis; thus $\zeta=\left(\rho^{2}+\zeta^{2}\right)^{1 / 2} \cos \omega, \rho=\zeta \tan \omega$. Let $P_{n}$ be the Legendre function of the first kind $P_{n}(\cos \omega)$. Since $F[(1+s) / 2,-s / 2 ; 1$; $\left.\sin ^{2} \omega\right]=P_{s}$ and $(2+s) \sin ^{2} \omega F\left[(3+s) / 2,-s / 2 ; 2 ; \sin ^{2} \omega\right]=2\left(P_{s}-\cos \omega P_{s+1}\right)$, we have

$$
M(s)=(2+s) c_{s} \iint_{A} k \sec ^{3} \omega P_{s} \zeta^{s} \rho d \rho d \zeta=c_{s} \int_{C} k \sec ^{2+s} \omega\left(P_{s}-\cos \omega P_{a+1}\right) \zeta^{2+s} d \zeta,
$$

where $(2+s) c_{s}=2 \pi \Gamma(1 / 2) \Gamma(1+s / 2) \Gamma(1-s / 2)$. The formula (41) solves the problem for a deeply buried dome. The parameters in this case (see Fig. 6) are: the three lengths $\bar{O}^{*} A_{j}=p_{j}, j=1,2,3$, the density contrasts $k_{1}=2 f \sigma_{1}, k_{2}=2 f \sigma_{2}$ and the angle $\omega$,

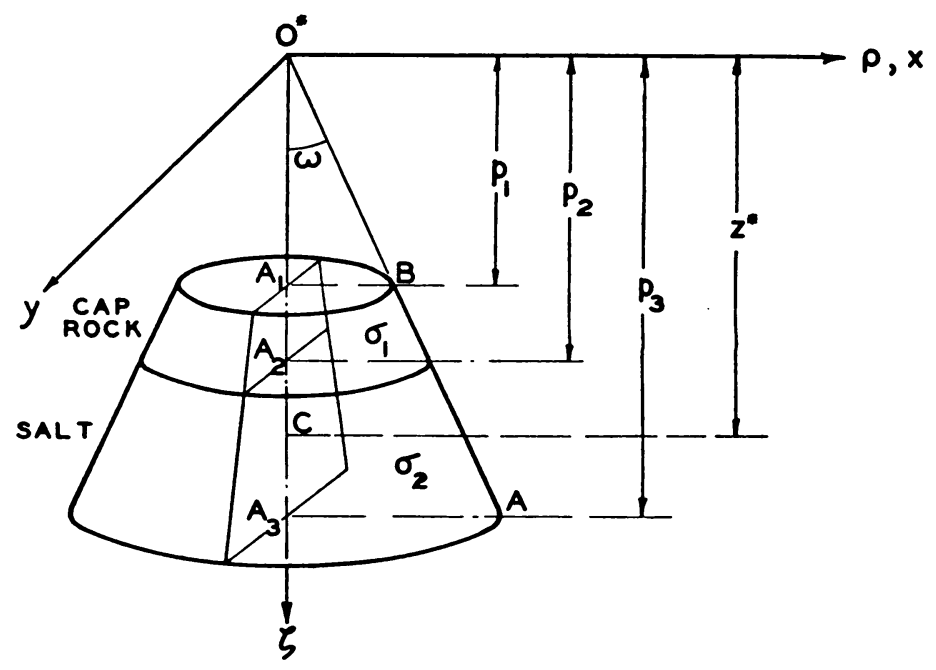

FIG. 6.

the slope of the dome's flanks. Instead of $p_{j}$ we use the ratios $r=p_{1} / p_{2}, q=p_{3} / p_{2}$ and write $p_{2}=p$. Also, $k=k_{2} / k_{1}$ this ratio being generally negative. The parameters $r<1$, $q>1, k$ verify the equation

$$
4 z^{*}\left[1-r^{3}+\left(q^{3}-1\right) k\right]=3\left[1-r^{4}+\left(q^{4}-1\right) k\right] p .
$$

In our case $\omega$ is constant in the second integral of (41) since only the integral 
along $\overline{B A}$ does not vanish. It is equal to $(3+s) k_{1} p^{3+s} \phi(s ; r, q, k)$, where $\phi(s ; r, q, k)$ $=1-r^{3+s}+k\left(q^{3+s}-1\right)$. Denoting $(3+s)^{-1} c_{s} \sec ^{2+s} \omega\left[P_{s}(\cos \omega)-\cos \omega P_{s+1}(\cos \omega)\right]$ by $f(s ; \omega)$, we have the explicit expression of the moment function $M(s)$

$$
M(s)=k_{1} p^{3+s} f(s ; \omega) \phi(s ; r, q, k) .
$$

The $f(s ; \omega)$ is easy to tabulate for eleven particular values of $s$, namely for $4 s=m$, where the integer $m$ verifies $-7 \leqq m \leqq 3$ since $-2<s<1$. If $4 s=m$ the function $P_{s}$ reduces to polynomials or to elliptic integrals $K$ and $E$. In fact, letting $\cos \omega=x$, we have $3 P_{-7 / 4}=3 P_{3 / 4}=P_{1 / 4}+2 x P_{-1 / 4}, P_{-5 / 4}=P_{1 / 4}, P_{-3 / 4}=P_{-1 / 4}, 5 P_{5 / 4}=6 x P_{1 / 4}-P_{-1 / 4}$, $21 P_{7 / 4}=10 x P_{1 / 4}+\left(20 x^{2}-9\right) P_{-1 / 4}$, where $\pi \cos (\omega / 4) P_{-1 / 4}=2[\cos (\omega / 2)]^{1 / 4} K$ and $\pi(\cos (\omega / 2)]^{1 / 4} P_{1 / 4}=2 \cos (\omega / 4)\left[4(E-K)+\left(1+\lambda^{2}\right) K\right]$ with the modulus $\lambda=\tan (\omega / 4)$.

Also $P_{-3 / 2}=P_{1 / 2}, 3 P_{3 / 2}=4 x P_{1 / 2}-P_{-1 / 2}$, where $P_{ \pm 1 / 2}$ are elliptic integrals too, but with modulus $\mu=\sin (\omega / 2)$, namely $\pi P_{-1 / 2}=2 K$ and $\pi P_{1 / 2}=4 E-2 K$. Transforming the experimental gravity map into the maps of the quantities $r^{s} D g(r)$ and integrating on these maps over $r \leqq R$, we obtain the reduced moment function $M^{*}(s)$ which differs from $M(s)$ only in the contribution of the infinite area $r \geqq R$. This contribution is computed with the aid of (36), and the reduction factor $\nu(s)$ in

$$
M(s)=M^{*}(s)\left[1+\nu(s)(p / R)^{1-\varepsilon}\right]
$$

is defined by $4(1-s) f(s ; \omega) \phi(s ; r, q, k) \nu(s)=3 f(0 ; \omega) \phi(1 ; r, q, k)$.

We consider equations of the general type $Q(s, t ; \omega, r, q, k)=N(s, t)$, where the function $Q$ of four unknowns $\omega, r, q, k$ is defined by $Q \equiv[f(s ; \omega) \phi(s)]^{-t}[f(t ; \omega) \phi(t)]^{s}$ - $[f(0 ; \omega) \phi(0)]^{t-s}$, the $\phi(s)$ denoting $\phi(s ; r, q, k)$. Thus the number $N(s, t)$ is to be calculated by the rule $N(s, t)=[M(s)]^{-\imath}[M(t)]^{\cdot}[M(0)]^{t-s}$. Its first value is obtained by using the reduced moment functions $M^{*}(s), M^{*}(t), M^{*}(0)$. With the aid of these first values $N^{*}$ we have to find first approximations for our parameters $\omega, r, q, k$. It is sufficient to form four equations of the type $Q=N$, giving to the orders $s$ and $t$ numerical values, for instance $s=-7 / 4$ and $t=-5 / 4,-3 / 2$ and $-1,-5 / 4$ and $-3 / 4,-1$ and $-1 / 2$. Solving such a system with the experimental data $N^{*}(-7 / 4$, $-5 / 4), N^{*}(-3 / 2,-1), N^{*}(-5 / 4,-3 / 4)$ and $N^{*}(-1,-1 / 2)$, we get the first approximate values for $\omega, r, q, k$. The depth $p$ is then found using the ratio $M^{*}(s) / M^{*}(0)$ for any value of $s$ (or $t$ ) and in practice the average value from many such determinations will be taken as $p$. Using the set of first approximations in (44), we improve the first values of the second members $N(s, t)$ in our system of four equations $Q=N$ and solving the improved system, we have second approximations for $\omega, r, q, k, p$. This procedure of successive approximations is continued until the stabilization of sequences. The depths $p_{1}=r p$ and $p_{3}=q p$ are found, as well as $k_{2}=k k_{1}$, since the value of $k_{1}$ can be obtained from the value of any $M(s)$. We observe that the interpretation yields both density contrasts $\sigma_{1}$ (cap-rock) and $\sigma_{2}$ (salt). The depth $z^{*}$ of the center of gravity obtained with the aid of $(42)$, compared with $z^{*}$ computed by $\left(23^{*}\right)$, gives a control. The control also is obtained with the aid of four other values of the order $s$ which were not used for the interpretation. We choose the negative values of $s$ in order to diminish the reduction factor in (44). It is interesting to add that the same method applies to the interpretation of $G$ - and $K$-maps, their moment functions $G(s), K(s)$ being expressed in terms of $M(s)$. In fact $G(s)=-(2+s) M(s-1)$ and 
$K(s)=c_{s}^{\prime} M(s-1)$ with $c_{s}^{\prime} \Gamma(1+s / 2) \Gamma(1 / 2-s / 2)=2 \Gamma(-s / 2) \Gamma(5 / 2+s / 2)$. For the interpretation of the magnetic maps we can use the same function $M(s)$ since the moment functions $A_{r}(s)$ and $Z(r)$ are given by $k_{2}(2+s) Z(s)=-2 I \sin \psi \cdot s K(s)$ and $k_{2} A_{r}(s)=-2 I \sin \psi \cdot(2+s) M(s)$.

In the general case, when the vertex of the cone is on the vertical of the point $O^{*}$ at the distance (unknown) $l$ above $(l>0)$ or below $(l<0)$ the ground, the moment function has a very complicated expression and is difficult to tabulate. In its place we use the integrals $D_{n}$ of the type

$$
D_{n}=l^{2 n-2} \iint_{P}\left(l^{2}+r^{2}\right)^{-(n+1 / 2)} D g(r) d S,
$$

and the interpretation yields all the seven parameters $k_{1}, k_{2}, p_{1}, p_{2}, p_{3}, \omega, l$, the angle $\omega$ being the slope of the dome's flanks. Lack of space does not permit the development of this general case.

Conclusion. The possibilities offered by the new method for quantitative interpretation of magnetic and gravitational anomalies we attempted to develop in this work seem to be very large. The mathematical tools, used in the proofs of the final interpretation rules, are not needed at all in practical applications. If the tables of functions used and the charts of auxiliary curves for the graphical solution of fundamental equations are calculated once for all and plotted, all that remains to be done in a particular case is the plotting of some auxiliary maps derived from the experimental data and the evaluation of some areas with the aid of a planimeter.

No use of average values in the interpretation is known to the author, with the exception of the work of $\mathrm{K}$. Jung, ${ }^{3}$ where only one integral, namely our $M(0)$, is defined and its value $2 f M$ is used together with remarkable values and distances.

${ }^{3}$ K. Jung, Zeit. f. Geophysik 13, 45-67 (1937). 